\title{
Antioxidant and antimicrobial studies of nanochitosan, carboxymethyl cellulose and graphene oxide binary and ternary composites
}

\author{
S. Sugashini, T. Gomathi* and P.N. Sudha* \\ Biomaterials Research Lab, PG and Research Department of Chemistry, D.K.M. College for \\ Women, Vellore, Tamil Nadu, India \\ (*Corresponding author Email: drparsu8@gmail.com (P.N. Sudha); drgoms1@gmail.com (T. \\ Gomathi))
}

\begin{abstract}
The present study aimed to prepare binary and ternary composites of nanochitosan (NCS), carboxymethyl cellulose (CMC) and graphene oxide (GO) for the biological and biomedical application. The prepared NCS/CMC, CMC/GO and NCS/CMC/GO composites were characterized using FTIR, XRD and SEM studies. The results revealed the suitability of the material for biological application with increased amorphous nature for cell adhesion. Antimicrobial studies were carried out against selected bacterial and fungal species. DPPH scavenging activity was done for the prepared biocomposites. NCS/CMC/GO ternary composite have the highest action against Bacillus sp $(21 \mathrm{~mm})$ followed by Klebsilla sp. (19 mm) and Proteus sp. (16 mm). For selected fungal species of Candida, Rhizopus and Aspergillus niger the ternary composites shows $28 \mathrm{~mm}, 26 \mathrm{~mm}$ and $24 \mathrm{~mm}$ inhibitory zone. The overall results exhibit the potential antioxidant and antimicrobial activity of the prepared composites.
\end{abstract}

Keywords: NCS/CMC/GO ternary composite, antibacterial studies, antifungal studies, antioxidant studies.

\section{Introduction}

Nanotechnology is a relatively new and rapidly developing technology that is gaining traction in a variety of fields as industry, agriculture, business, medicine and public health [1]. It will integrate the nanoscale structures into large components and systems by keeping their benefits [2,3].A variety of nanomaterials have been produced and investigated for primary food packaging nowadays. Its major function is to improve the material's mechanical, barrier, antibacterial, and thermal properties [4]. Biopolymers in this regard acts as good antimicrobial agents. Biopolymers due to its low toxicity, biodegradability, and stability it can be used in a variety of pharmacological and medicinal applications.

Especially, chitosan biopolymer due to its $\mathrm{OH}$ and $\mathrm{NH}$ functionality it possess the properties including biodegradability, non-toxicity, high elasticity, good adhesion and sorption $[5,6]$. These properties made chitosan (CS) as a promising material for antimicrobial applications [7, 8]. Thus, in the present work chitosan biopolymer was converted into its nanoform via ionic gelation method and mixed with carboxymethyl cellulose and graphene oxide to improve its biological properties.

Carboxymethyl cellulose (CMC) is an anionic polymer that is linear, long-chained, and water soluble [9]. Due to its high transparency, good film-forming property, large mechanical 
strength, non-toxicity, and biodegradability, (CMC) is widely used as a packaging material (for films and coatings), medicine, flocculating agent, chelating agent, emulsifier, thickening agent, water-retaining agent, and sizing agent [10]. In this context, graphene oxide (GO) is one of the most appealing nanofillers in polymer nanocomposite technology because it improves and enhances the mechanical, thermal, and electrical properties of many nanocomposites, which could lead to novel solutions for a variety of applications [11].

GO has antibacterial properties, which has sparked interest in developing GO-based antimicrobial surfaces $[12,13]$. The protection of hazardous biocides from being released was a key component in the design of these antimicrobial GO surfaces. It can induce bacterial cells to adhere together, causing cell integrity to be compromised and loss of cell viability, limiting bacterial growth on the surface [14 - 16]. As a result, these materials have significant applications in biomedicine, marine transportation, membrane-based water treatment, and heat exchangers $[17,18]$.

Hence, in the present work, the polymer composites of Nanochitosan (NCS), Carboxymethyl cellulose (CMC), and Graphene oxide (GO) in the form of binary and ternary composites were prepared by solution casting method. The prepared composites were subjected to FTIR, XRD and SEM studies and evaluated its antioxidant and antimicrobial properties.

\section{Material and Methods}

\section{Chemicals and reagents}

Chitosan (deacetylation 92\% and MW 120,000) was obtained from India Sea Foods, Cochin, India, while AR grade tripolyphosphate and acetic acid were obtained from Finar chemicals, Ahmadabad, and Thomas Bakers chemicals Pvt. Ltd., Mumbai, respectively, and used without further purification. Carboxymethyl cellulose (CMC)was purchased from Molychem, Mumbai. Graphite fine power extremely pure was procured from Sisco Research Laboratories, Pvt. Ltd., Chennai.

\section{Preparation of Nanochitosan}

The nanochitosan was made using the sol-gel process, which involved dissolving 1 gof chitosan in $100 \mathrm{~mL}$ of $2 \%$ acetic acid. TPP solution (0.8 $\mathrm{g}$ in $107 \mathrm{ml} \mathrm{DD}$ water) was progressively added to this, stirring constantly. Nanochitosan was obtained as a suspension. The supernatant was discarded, and the nanochitosan was air dried for usage and analysis (19).

\section{Preparation of Graphene oxide (GO)}

The Hummers method was used to functionalize the graphite (20). $50 \mathrm{~mL}$ of cold $\mathrm{H}_{2} \mathrm{SO}_{4}$ (cooled under water bath till it reaches ice cold temperature of $0^{\circ} \mathrm{C}$ ) was used to dissolve $1 \mathrm{~g}$ of graphite and $0.5 \mathrm{~g}$ of $\mathrm{NaNO}_{3}$. For 30 minutes, the mixture is stirred continuously. About $4 \mathrm{~g}$ of $\mathrm{KMnO}_{4}$ was gently added to the aforesaid mixture under steady stirring for further oxidation. The temperature was kept below $20^{\circ} \mathrm{C}$ for the $\mathrm{KMnO}_{4}$ addition. By placing the beaker in an ice bath for about 1 hour, the temperature of the reaction mixture was decreased to ice cold. The addition of $\mathrm{KMnO}_{4}$ was done slowly and carefully with control. The ice bath was then removed, and the reaction was allowed to continue by swirling the solution for 30 minutes at $35^{\circ} \mathrm{C}$ with magnetic stirring.

$50 \mathrm{~mL}$ DD water was progressively added to this combination. The reaction was then continued at $98^{\circ} \mathrm{C}$ for 20 minutes, followed by the addition of $200 \mathrm{~mL}$ of DD water and $5 \mathrm{~mL}$ of 
$\mathrm{H}_{2} \mathrm{O}_{2}(30 \%)$ to remove any remaining $\mathrm{KMnO}_{4}$ and $\mathrm{MnO}_{2}$. For the final purification of graphite oxide, the resulting mixture was filtered and washed with DD water and a 5 percent $\mathrm{HCl}$ solution $(50 \mathrm{~mL})$. The graphene oxide $(\mathrm{GO})$ powder was obtained after filtration and drying under vacuum at $70^{\circ} \mathrm{C}$.

\section{Preparation of Binary ternary composites}

$1 \mathrm{~g}$ Under magnetic stirring, $1 \mathrm{~g}$ of carboxymethyl cellulose dissolved in water and $1 \mathrm{~g}$ of nanochitosan dispersed in water were mixed for about 30 minutes. The 1:1 CMC/NCS binary compound was poured into petridish and allowed to air dry. The CMC/GO binary composite was prepared using the same approach.

\section{Preparation of Ternary composites}

$1 \mathrm{~g}$ of nanochitosan was combined with $1 \mathrm{~g}$ of $\mathrm{CMC}$ dissolved in water to make the NCS/CMC/GO ternary composite. This was followed by the addition of $0.5 \mathrm{~g}$ of graphene oxide. The solution was well agitated for 30 minutes with moderate agitation before being placed into a petridish and allowed to air dry.

\section{Characterization}

Fourier Thermo NicoletAVATAR 330 spectrophotometer was used to perform FTIR spectrum studies of the produced blends in the $4000-400 \mathrm{~cm}^{-1}$ wave length region, utilising the $\mathrm{KBr}$ pellet method. $\mathrm{X}$-ray diffraction (XRD) patterns were investigated utilising a Ni-filtered $\mathrm{Cu}$ $\mathrm{K} X$-ray radiation source and an X-ray powder diffractometer (XRD -SHIMADZU XD - D1). At a scanning rate of $50 \mathrm{~min}^{-1}$, the relative intensities were obtained in the range of $5^{\circ}-90^{\circ}$. The ratio of crystalline area to total area of the peaks can be used to calculate the percentage of crystallinity $(\mathrm{Xc})$. The composite was sliced into various sizes and wiped with a thin gold palladium layer by a sputter coater unit (VG - microtech, UCK field, UK) for examination, and the cross section topography was examined with a Cambridge stereoscan 440 scanning electron microscope TESCAN VEGA3 SBH.

\section{Antimicrobial activity binary and ternary composites}

The antibacterial activity of NCS/CMC and CMC/GO binary composites, as well as NCS/CMC/GO (1:1:0.5) ternary composites, was investigated using the diffusion method on Muller Hinton Agar (MHA) medium with three bacterial strains: Bacillus sp., Klebsilla sp., and Proteus sp. gram positive and gram negative bacteria. Bacillus sp., Klebsilla sp., and Proteus sp. were inoculated on Muller Hinton Agar (MHA) medium and spread uniformly in Petri plate using sterile spreader. After the MHA medium had solidified, a small amount of the binary composites were placed on different cultured agar plates, which were then incubated on individual racks for 24 hours at $37^{\circ} \mathrm{C}$. The diameter of the zone of inhibition grown around the sample against the test microorganism was measured using the rules to assess the antibacterial activities of the prepared sample. Similarly the antifungal activity was done using the pathogenic fungi such as Candidasp, Rhizopussp, and Aspergillus Niger sp on agar plates the samples.

\section{Antioxidant Activity of binary and ternary composites}

The DPPH scavenging experiment was used to test the composite's antioxidant properties. In antioxidant activity research, DPPH radical scavenging activity is a common test. The obtained composite's radical scavenging ability was tested by mixing (20-200 g/mL) with 4 
$\mathrm{mL}$ DPPH (0.004\% in methanol) and incubating at room temperature for 30 minutes at the absorbance $517 \mathrm{~nm}$. When the colour changes, the absorbance drops as the DPPH is scavenged by the sample by hydrogen atom donation to create a stable DPPH complex. As a control, a DPPH solution with no sample is used.

The percentage of inhibition (I \%) of free radical production from DPPH was calculated by using the following equation

$$
\% \text { of Inhibition }=\frac{A_{c}-A_{s}}{A_{c}} \times 100
$$

where, Ac - Absorbance of the control and As - Absorbance of the sample solution.

\section{Results and Discussions FTIR studies}

Hydrogen bonding and miscibility of the composite can be analyzed using the FTIR spectroscopy technique, which determines the functional group present in a molecule as well as the chemical and structural bond information [21]. The FTIR spectrum of the NCS/CMC binary composite, CMC/GO binary composite, and NCS/CMC/GO ternary composite is shown in Figures 1-3. The existence of $\mathrm{OH}$ stretching and $\mathrm{NH}$ stretching vibrations in the $\mathrm{H}$-bond formation is shown by a broad band at $3444.84 \mathrm{~cm}^{-1}, 3427.51 \mathrm{~cm}^{-1}$, and $3439 \mathrm{~cm}^{-1}$ in the binary and ternary composites. Table 1 shows the numerous bands of polymer composites that reflect the respective functional group.

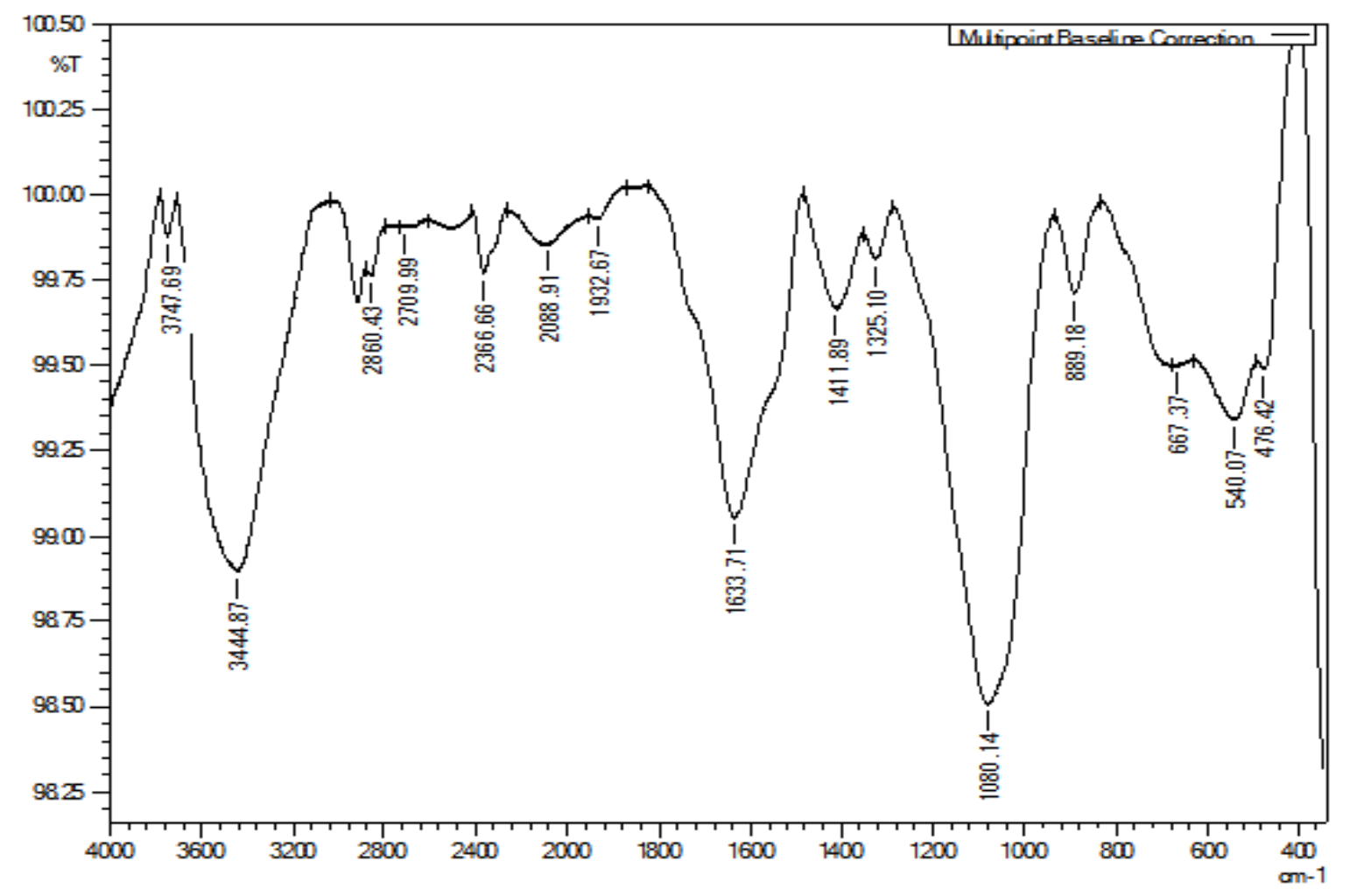

Figure 1: FTIR spectrum of NCS/CMC binary composite 


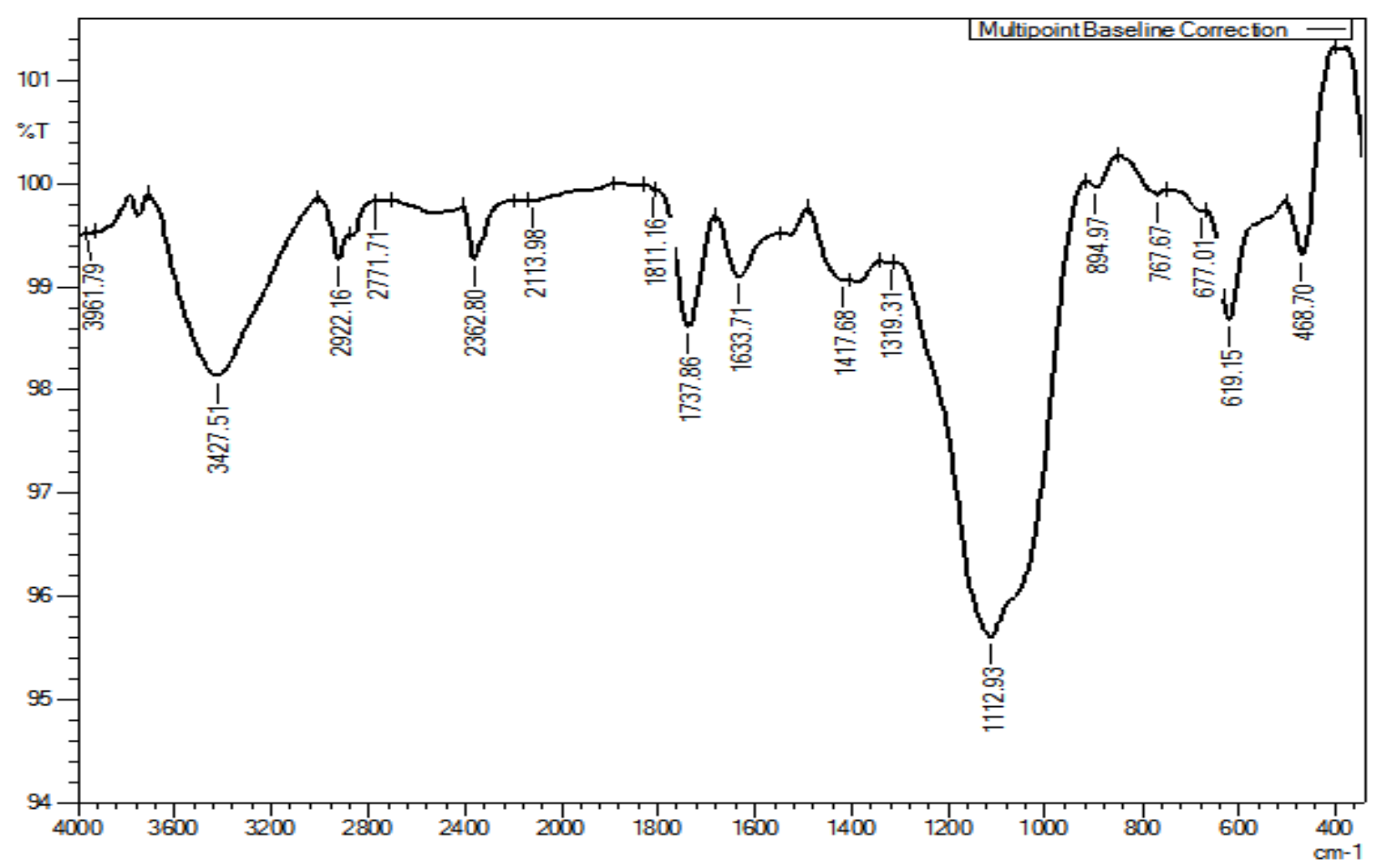

Figure 2: FTIR spectrum of CMC/GO binary composite

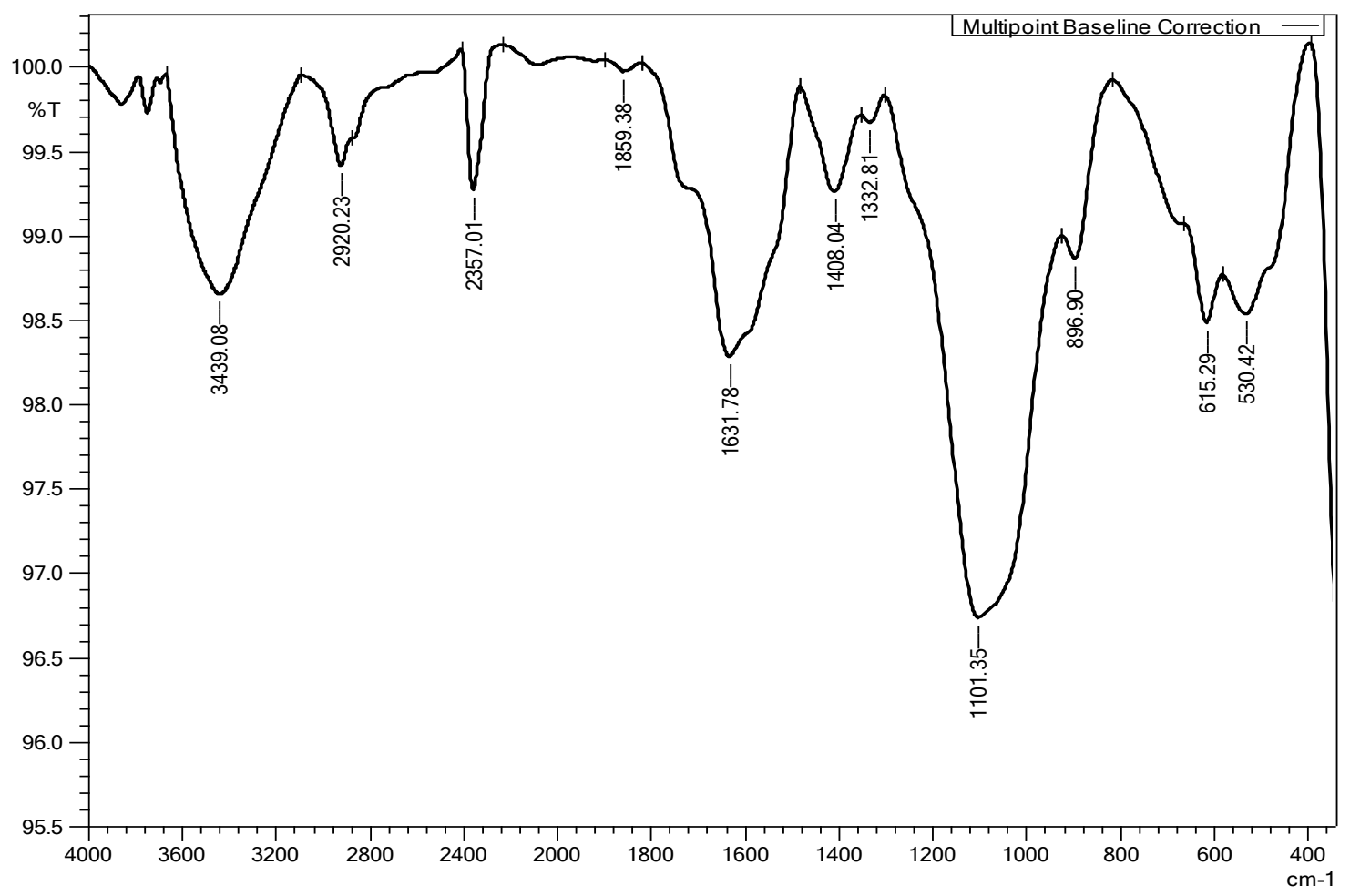

Figure 3: FTIR spectrum NCS /CMC/GO ternary composite 
Table 1: FTIR details of NCS, CMC and GO composites

\begin{tabular}{|c|c|c|l|}
\hline \multicolumn{3}{|c|}{ Wavenumber $\left(\mathbf{c m}^{-1}\right)$} & \multicolumn{1}{|c|}{ Functional Group } \\
composite & $\begin{array}{c}\text { CMC/GO binary } \\
\text { composite }\end{array}$ & $\begin{array}{c}\text { NCS/CMC/GO } \\
\text { ternary composite }\end{array}$ & \\
\hline 3444 & 3427 & 3439 & $\begin{array}{l}\text { OH Stretching and NH } \\
\text { stretching vibration }\end{array}$ \\
\hline 2860 & 2922 & 2920 & Asymmetric CH Stretching $^{+}$ \\
\hline 2366 & 2362 & 2357,1859 & $\mathrm{NH}_{3}{ }^{+}$Stretching \\
\hline 1633 & 1633 & 1631 & $\begin{array}{l}\text { C=O Stretching } \\
\text { (Amide Group) }\end{array}$ \\
\hline 1411 & 1417 & 1408 & CH bending \\
\hline 1325 & 1319 & 1332 & C-H and OH bending \\
\hline 1080.14 & 1112.93 & 1101 & C-N and P=O stretching \\
\hline 889.18 & 894.97 & 896 & C-O-Clinkage \\
\hline
\end{tabular}

The bands found in the binary and ternary composites at $2366 \mathrm{~cm}^{-1}, 2362 \mathrm{~cm}^{-1}$, and 2920 $\mathrm{cm}^{-1}$ confirm the existence of protonated $\mathrm{NH}_{3}{ }^{+}$groups, which would have played a role in the production of nanochitosan via electrostatic contact. In the binary and ternary composites, carbonyl stretching (amide I) is found at $1633.71 \mathrm{~cm}^{-1}, 1633.71 \mathrm{~cm}^{-1}$, and $1631 \mathrm{~cm}^{-1}$, respectively. The FTIR spectra of NCS/CMC, CMC/GO, and NCS/CMC/GO composites shows a matching band shift and intensity shift, indicating that the composites were formed through strong interactions between the polymers.

\section{XRD studies}

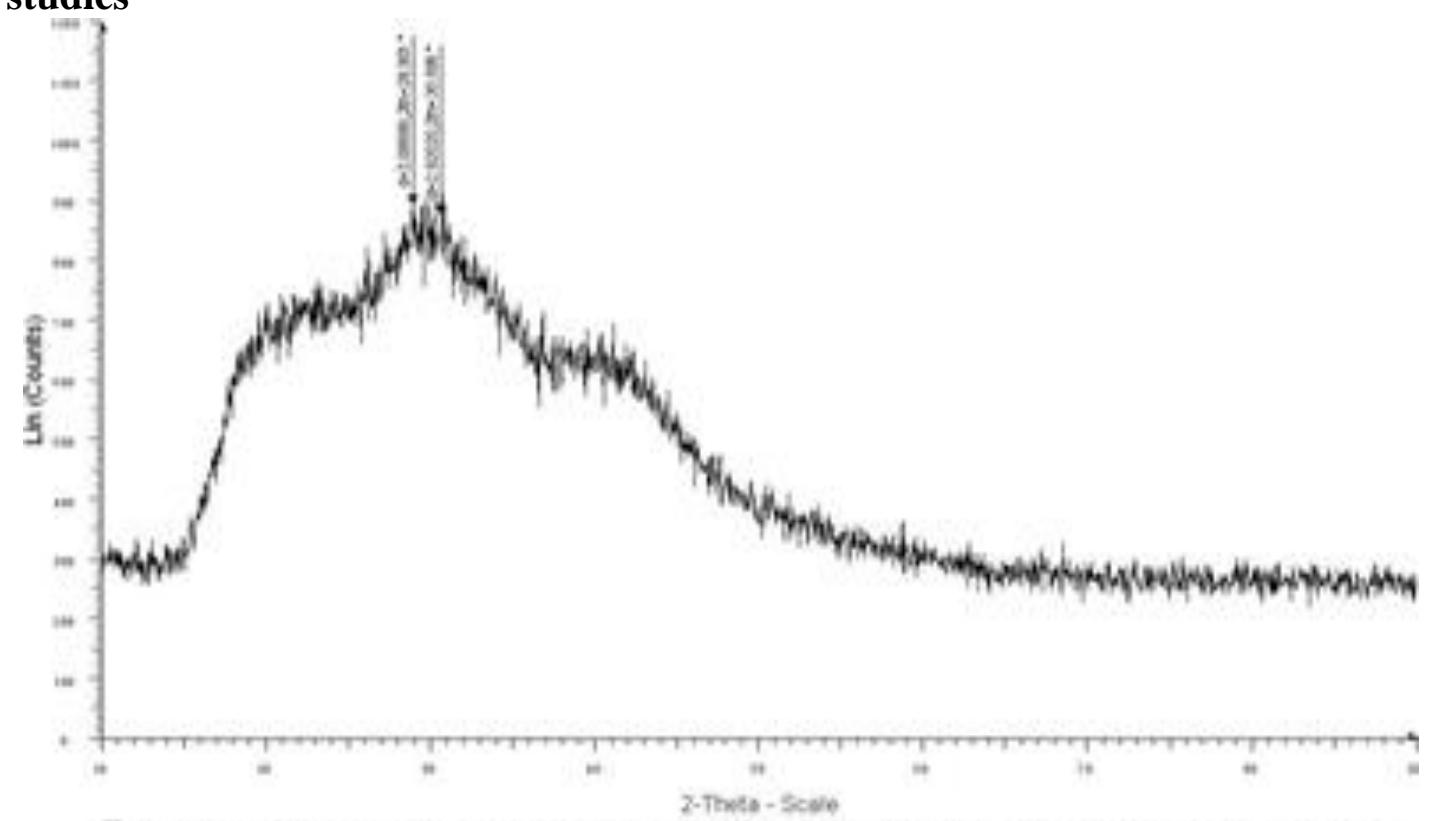

Figure 4: XRD pattern of NCS/CMC binary composite 


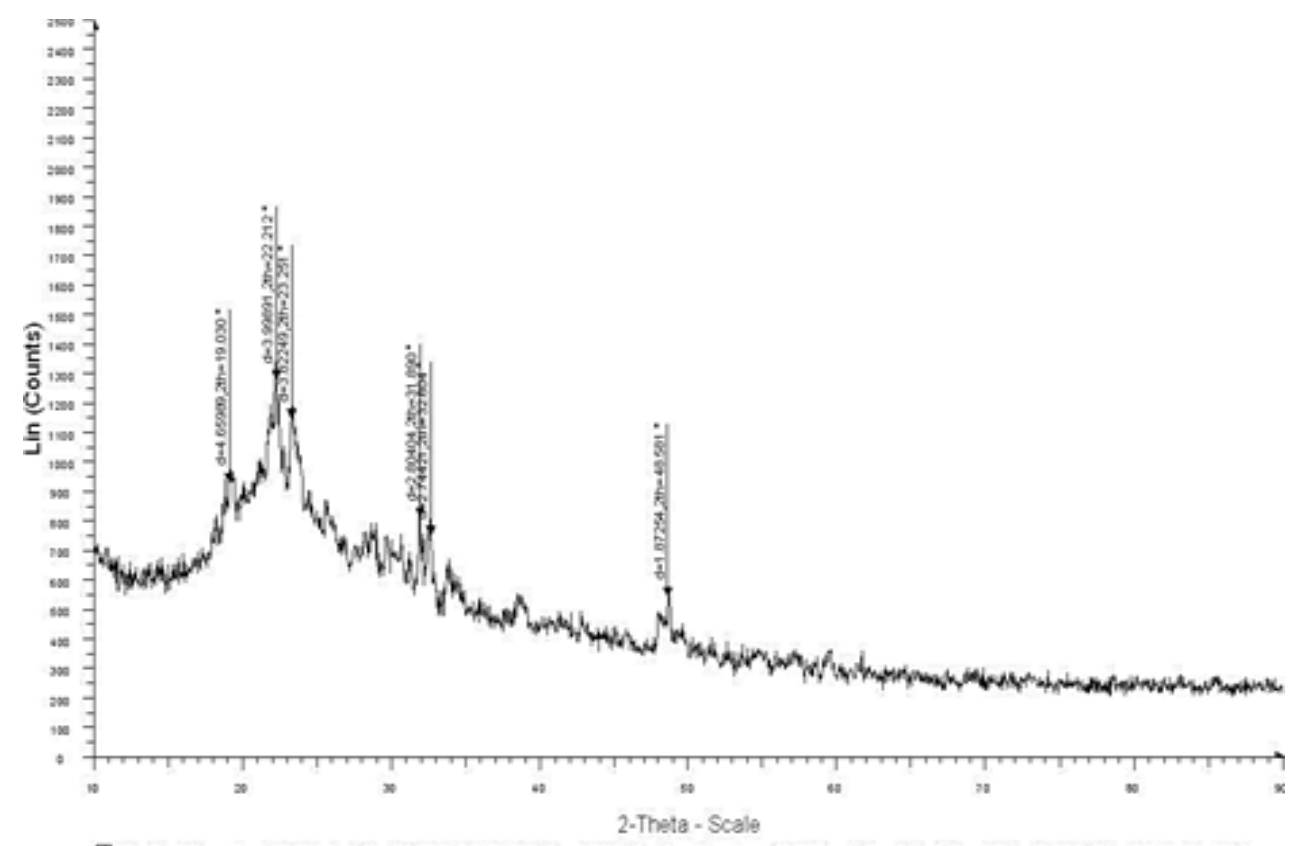

Figure 5: XRD pattern of CMC/GO binary composite

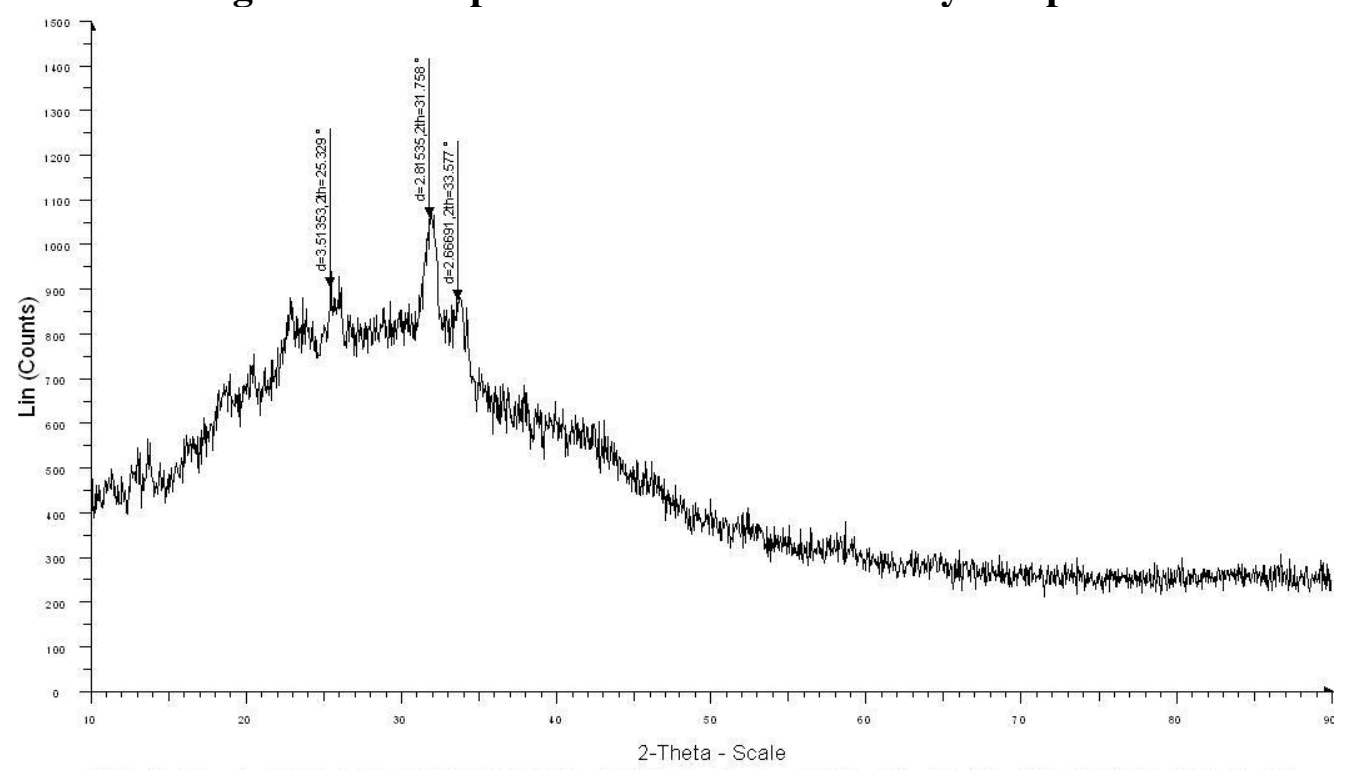

Figure 6: XRD spectrum NCS/CMC/GO ternary composite

The XRD pattern for the binary and ternary composites of NCS, CMC, and GO is shown in Figures 4-6. The amorphous nature of the produced composites is confirmed by the XRD pattern's broad feature. The binary composites NCS/CMC and CMC/GO have two short broad peaks in their $\mathrm{XRD}$ patterns $2 \theta=28^{\circ}$ and $30^{\circ}$ and $2 \theta=19^{\circ}$ and $22^{\circ}$ respectively, while the ternary composite displayed broad peaks $2 \theta=25^{\circ}$ and $27^{\circ}$, indicating the amorphous nature of the composite. The XRD pattern of nanochitosan composites shows a single well-defined peak and a shoulder peak with a shift in peak position, indicating modification of polymers to form binary and ternary composite [22]. 


\section{SEM}

SEM is widely used to investigate both the surface morphology of materials and the biological response to them. Surface properties have a huge impact on whether a material device succeeds or fail, implying the necessity and need for accurate characterization of the material surface [23]. Figure 7a depicts the SEM morphology of the NCS/CMC binary composite, which is very favourable for since the surface area has been substantially enhanced while a very stable composite has been formed. The porous aspect of the binary composite is owing to the inclusion of GO, which increases the stiff structure of the material, as shown in Figure 7b. Figure 7c shows a SEM picture of the NCS/CMC/GO composite, which reveals a rough surface and an open pore structure with distinct needle walls and a high degree of pore interconnectivity in the created ternary composite material.
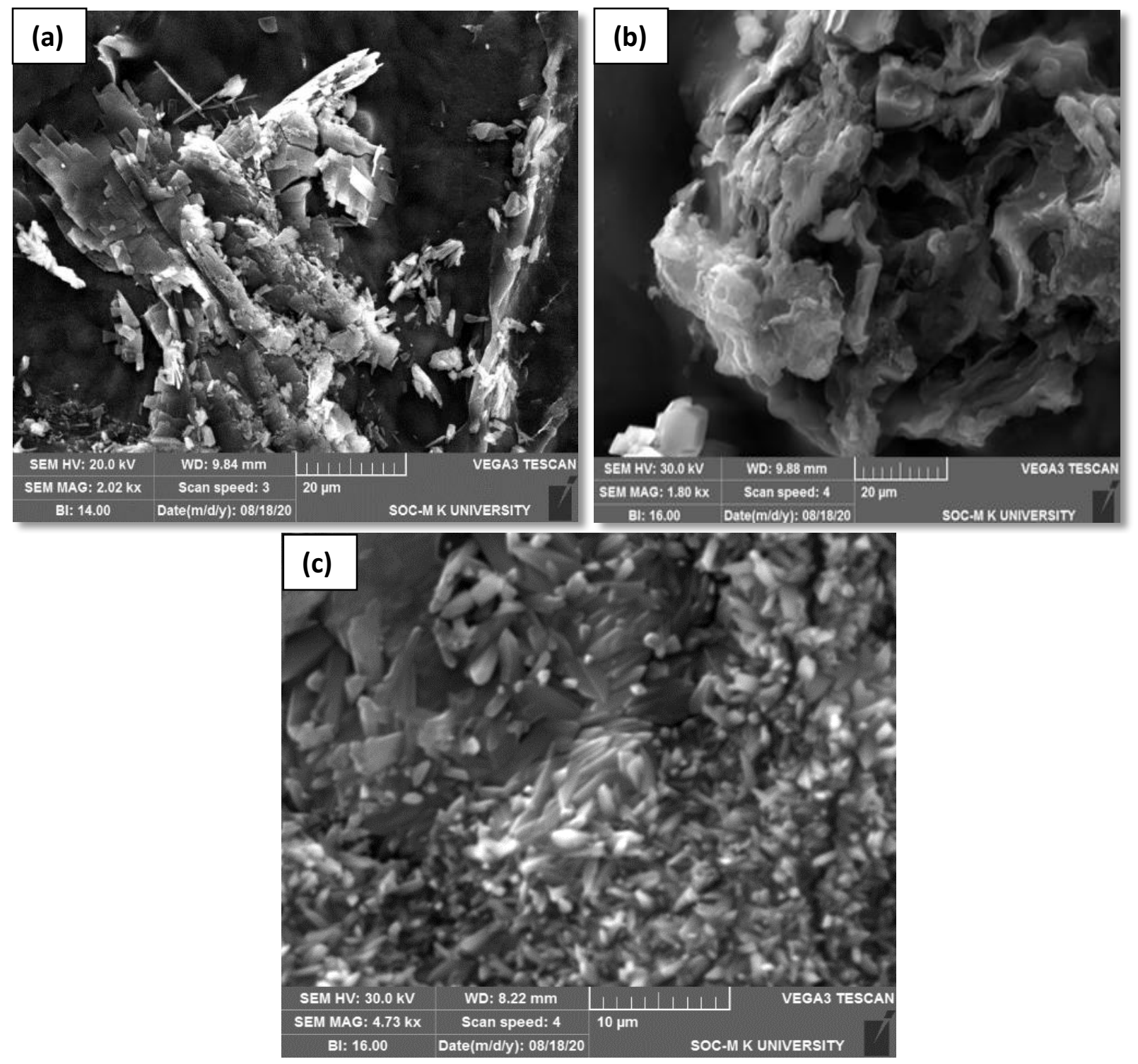

Figure 7: SEM images of (a) NCS/CMC; (b) CMC/GO and (c) NCS/CMC/ GO composites 
Antimicrobial study

Antibacterial activity

Table 2: Antibacterial Activity of binary composites and ternary composite

\begin{tabular}{|l|c|c|c|c|}
\hline \multirow{2}{*}{ Organism (Bacteria) } & \multicolumn{4}{|c|}{ Diameter of zone of Inhibition (cm) } \\
\cline { 2 - 5 } & $\begin{array}{l}\text { Control } \\
\text { Ciprofloxacin }\end{array}$ & NCS/CMC & CMC/GO & $\begin{array}{c}\text { NCS/CMC/GO } \\
(\mathbf{1 : 1 : 0 . 5 )}\end{array}$ \\
\hline Bacillus species & 22 & 19 & 20 & 21 \\
\hline Klebsilla species & 17 & 18 & 19 & 20 \\
\hline Proteus species & 15 & 17 & 16 & 18 \\
\hline
\end{tabular}

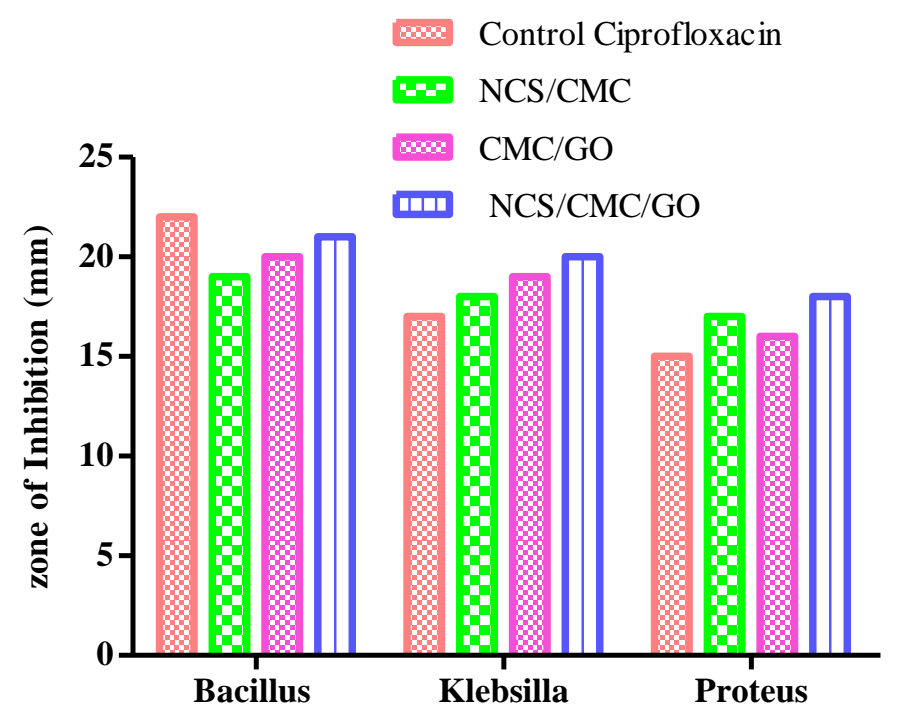

Figure 8: Antibacterial activity of binary composites and ternary composites

The antibacterial activity of the prepared NCS/CMC and CMC/GO binary composite and $\mathrm{NCS} / \mathrm{CMC} / \mathrm{GO}$ ternary composites was tested using agar disk diffusion method and the results are shown in Figure 8 and Table 2. Ciprofloxacin was used as control. The zone of inhibition values revealed that the prepared NCS/CMC, CMC/GO and NCS/CMC/GO composites could inhibit the growth of both gram-negative and gram-positive bacteria species. These results revealed the existence of strong electrostatic interaction between the prepared biocomposite and the bacterial cell wall. Due to this interaction between nanochitosan composite and lipopolysaccharides (gram -ve) and teichoic acids (gram +ve) there will be the blockage of intra/extracellular exchanges and disruption in bacterial cell wall resulting in the leakage of cytoplasmic content. In comparison between selected bacterial strains, NCS/CMC/GO had the highest action against Bacillus sp $(21 \mathrm{~mm})$ followed by Klebsilla sp. $(19 \mathrm{~mm})$ and Proteus sp. $(16 \mathrm{~mm})$. It's possible that the discrepancy is due to their differing cell walls [24].

\section{Antifungal Activity}

$\mathrm{NCS} / \mathrm{CMC}, \mathrm{CMC} / \mathrm{GO}$ and $\mathrm{NCS} / \mathrm{CMC} / \mathrm{GO}$ biocomposites was investigated against Rhizopus sp., Aspergillus niger, and Candida sp. The measured zone of inhibition was given in the table 3 and figure 9 . 
Table 3: Antifungal Activity of binary composites and ternary composite

\begin{tabular}{|l|c|c|c|c|}
\hline \multirow{2}{*}{$\begin{array}{c}\text { Organism } \\
\text { (Fungi) }\end{array}$} & $\begin{array}{l}\text { Control } \\
\text { Amphotericin }\end{array}$ & CMC/NCS & CMC/GO & $\begin{array}{c}\text { NCS/CMC/GO } \\
(\mathbf{1 : 1 : 0 . 5 )}\end{array}$ \\
\hline Candida & 24 & 19 & 20 & 28 \\
\hline Rhizopus & 23 & 18 & 19 & 26 \\
\hline Aspergillus niger & 22 & 17 & 18 & 24 \\
\hline
\end{tabular}

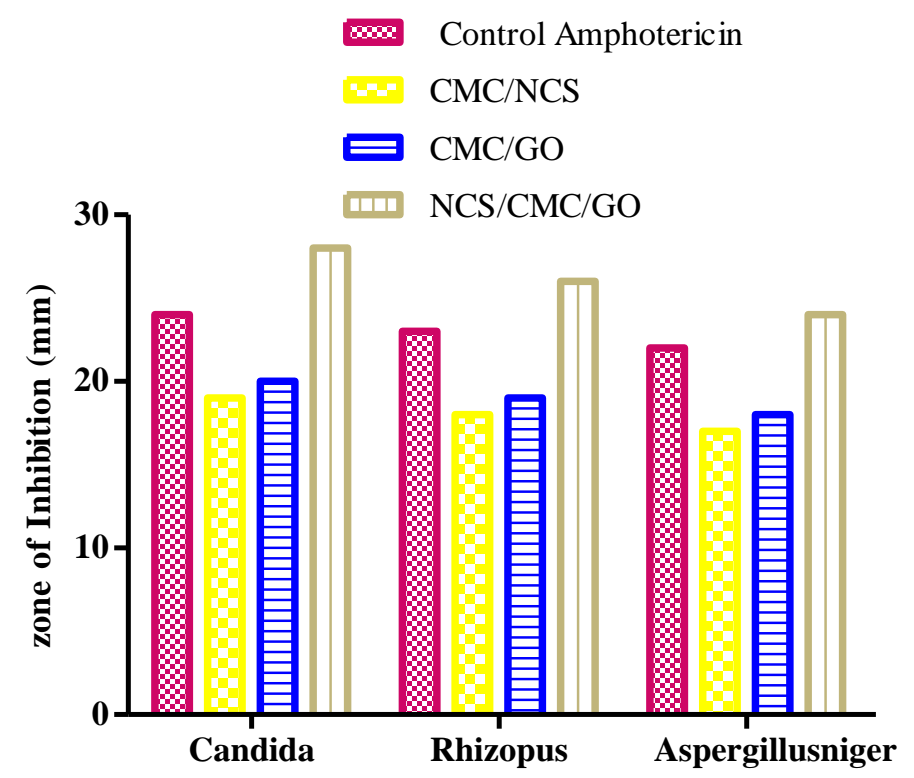

Figure 9: Antifungal Activity of binary composites and ternary composite

Higher inhibitory zone was recorded against Aspergillus niger, candida sp. and Rhizopus sp. NCS/CMC/GO biocomposite when compared with standard control Amphotericin. This superior antifungal activity was due to the change in physiological $\mathrm{pH}$ in the microbial cell and the formation of water proof layer. Hence, the prevention of transportation of food materials for the survival of microbial cells has taken place.

\section{Antioxidant activity of Binary and ternary composites}

The antioxidant activity of prepared binary composites and ternary composite was studied using the DPPH radical scavenging method. The DPPH radical shows strong absorption with NCS/CMC, $\mathrm{CMC} / \mathrm{GO}$ and NCS/CMC/GO biocomposites at $517 \mathrm{~nm}$ using spectrophotocalorimeter for the existence of odd electrons. While DPPH free radical scavenging activity is used to assess substances for their ability to behave as free radical scavengers. The antioxidant activity of polysaccharides has been connected to the kind of monosaccharide, uronic acid content, sulfation degree, glycosidic linkage, and molecular weight [25].

The presence of $\mathrm{OH}$ and $\mathrm{NH}$ functional groups in NCS, CMC and GO exhibits radical scavenging activity and can protect a variety of biomolecular target molecules from oxidation [26]. The increase in antioxidant of ternary composite than binary was mainly depends on the 
hydrogen donation capability of polymer composites. As shows in figure binary composites and ternary composite of exhibits good antioxidant activity

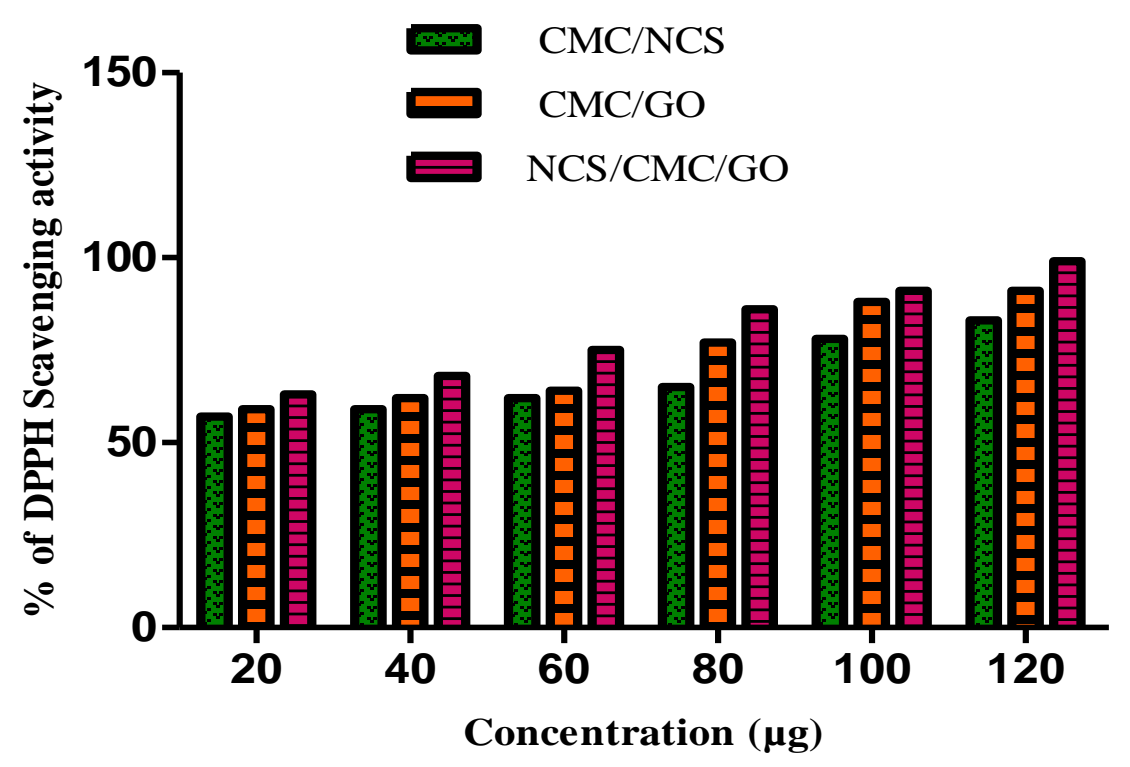

Figure 10: Antioxidant activity of prepared binary and ternary composite

\section{Conclusion}

Nanochitosan/Carboxymethyl cellulose, Carboxymethyl cellulose/Graphene oxide, and Nanochitosan/Carboxymethyl cellulose/Graphene oxide composites were effectively generated and characterised utilising FTIR, XRD, and SEM analyses in the current work. The physical and chemical interaction between the polymer composites taken is shown by the FTIR spectrum. XRD studies reveal a decrease in crystalline nature and an increase in amorphous character in ternary composites, which can improve mechanical strength over binary composites. SEM image indicate the composites was rough surface better than ternary composite very stable composite. The results of antimicrobial studies showed the potentate antimicrobial effect of binary composites NCS/CMC, CMC/GO and ternary composite NCS/CMC/GO against selected bacterial and fungal species. Thus, suggesting that it can be used for future application food packing material and wound healing applications.

\section{References}

[1] N. M. Noah., Design and Synthesis of Nanostructured Materials for Sensor Applications, Journal of Nanomaterials. (2020) 1-20. doi:10.1155/2020/8855321.

[2] J. Jeevanandam, A. Barhoum, Y. S. Chan,A.Dufresne, M. K. Danquah, Review on nanoparticles and nanostructured materials: history, sources, toxicity and regulations. Beilstein Journal of Nanotechnology. 9 (2018) 1050-1074. doi:10.3762/bjnano.9.98.

[3] I. Yunus, S.Harwin, Kurniawan, A. D. Adityawarman,A.Indarto, Nanotechnologies in water and air pollution treatment, Environmental Technology Reviews.1(1) (2012) 136148. doi:10.1080/21622515.2012.733966.

[4] J. K.Patra, G. Das, L. F.Fraceto,E. V. R. Campos, M. del P. Rodriguez-Torres, LAcostaTorres, H.-S. Shin, Nano based drug delivery systems: recent developments and future 
prospects, Journal of Nanobiotechnology. (2018) 16(1). doi:10.1186/s12951-018-03928.

[5] Haleema,Saleem and Syed,Javaid Zaidi, Developments in the Application of Nanomaterials for Water Treatment and Their Impact on the Environment, Nanomaterials. 10 (2020) 1764. doi: 10.3390/nano10091764.

[6] G. Hojnik Podrepšek, Z. Knez, M. Leitgeb. Development of Chitosan Functionalized Magnetic Nanoparticles with Bioactive Compounds. Nanomaterials (Basel). 2020;10(10):1913. Published 2020 Sep 25. doi:10.3390/nano10101913.

[7] N. M.J. F. Alves, Mano, Chitosan derivatives obtained by chemical modifications for biomedical and environmental applications, International Journal of Biological Macromolecules. 43(5) (2008) 401-414. doi:10.1016/j.ijbiomac.09.007.

[8] L. P. Gomes, V. M. F. Paschoalin, Del Aguila, E. M. Rev, Chitosan Nanoparticles, Production, Physicochemical Characteristics and NutraceuticalApplications.Virtual Quim. ,9(1) (2016) 387-409.

[9] Barbosa, Coutinho, Costa Lima,Reis, Marine Polysaccharides in Pharmaceutical Applications: Fucoidan and Chitosan as Key Players in the Drug Delivery Match Field. Marine Drugs, 17(12) (2019) 654. doi:10.3390/md17120654.

[10] Su.Shi, M. Peter, Kang, Systemic Review of Biodegradable Nanomaterials in Nanomedicine, Nanomaterials. 10 (2020) 656. doi:10.3390/nano10040656

[11] R. Prasad, A. Bhattacharyya, Q. D. Nguyen, Nanotechnology in Sustainable Agriculture: Recent Developments, Challenges, and Perspectives, Frontiers in Microbiology. (2017) 8. doi:10.3389/fmicb.01014.

[12] C.Arancibia, R.Navarro-Lisboa, R. N. Zúñiga,S. Matiacevich, Application of CMC as Thickener on Nanoemulsions Based on Olive Oil: Physical Properties and Stability, International Journal of Polymer Science. (2016) 1-10. doi:10.1155/2016/6280581.

[13] L. F. Zemljič, M.Bračič, T. Ristić, I. Šauperl, S.Strnad, Z.Peršin, Functionalization of Polymer Materials for Medical Applications Using Chitosan Nanolayers, Polymeric Nanomaterials in Nanotherapeutics. (2019) 333-358. doi:10.1016/b978-0-12-8139325.00009-1

[14] T. Maver, L. Gradišnik, D.M.Smrke, K. StanaKleinschek, U.Maver, Systematic Evaluation of a Diclofenac-Loaded Carboxymethyl Cellulose-Based Wound Dressing and Its Release Performance with Changing $p H$ and Temperature, AAPS PharmSciTech. 20(1) (2019). doi:10.1208/s12249-08-1236-4.

[15] Hui. Wang, Letian. Wang, Shanyu. Meng Lin. Hanxue Lin, Melanie.Correll, Zhaohui Tong, Nanocomposite of Graphene Oxide Encapsulated in Polymethylmethacrylate (PMMA) Pre-Modification Synthesis and Latex Stability, J. Compos. Sci. 4 (2020) 118. doi:10.3390/jcs4030118

[16] L. Xu, J.Teng, Li. Huang, H.-D. Xu, Y.Li,Hydrophobic Graphene Oxide as a Promising Barrier of Water Vapor for Regenerated Cellulose Nanocomposite Films, ACS Omega. 4(1) (2019) 509-517. doi:10.1021/acsomega.8b0286.

[17] S.S.An, S.Y. Wu, J. Hulme, Current applications of graphene oxide in nanomedicine, International Journal of Nanomedicine. 9 (2015). doi:10.2147/ijn.s88285.

[18] Ali.Ammar, M. Abdullah, Al-Enizi.Mariam,AlAli,AlMaadeed, Karim. Alamgir, Influence of graphene oxide on mechanical, morphological, barrier, and electrical properties of polymer membranes, Arabian Journal of Chemistry.(2015), http://dx.doi.org/10.1016/j.arabjc.2015.07.006 
[19] D.M. Warsinger, S.Chakraborty, E.W. Tow, M.H.Plumlee, C. Bellona, S.Loutatidou, J.H.Lienhard, A review of polymeric membranes and processes for potable water reuse, Progress in Polymer Science.81 (2018) 209-237. doi:10.1016/j.progpolymsci.

[20] A .Ammar, A.M. Al-Enizi, M.A. AlMaadeed, A. Karim, Influence of graphene oxide on mechanical, morphological, barrier, and electrical properties of polymer membranes, Arabian Journal of Chemistry. 9(2) (2016) 274-286. doi:10.1016/j.arabj.07.006

[21] Madhuri. Sharon, Farha. Modi, Sharon.Maheshwar, Titania based nanocomposites as a photocatalyst A review, AIMS Materials Science. 3(3) (2016) 1236-1254. DOI: 10.3934/matersci.3.1236.

[22] Zhang. Fubao, Wang. Xianming, Liu. Haonan, Liu. Chunli, Yong Wan, Long.Yunze, Cai. Zhongyu, Recent Advances and Applications of Semiconductor Photocatalytic Technology, Appl. Sci. 9 (2019) 2489. doi:10.3390/app9122489

[23] V. Alexander, Héctor, Vorontsov, Valdés, G. Panagiotis, Yaron. Smirnioti, Yaron.Paz. Recent Advancements in the Understanding of the Surface Chemistry in TiO2 Photocatalysis. Surfaces.3 (2020) 72-92. doi: 10.3390/surfaces3010008.

[24] Wei. Zhu, Xueliang,Jianb.Fangjun, Liu. Feng, You . Chu Yao,. Preparation of Chitosan-Graphene Oxide Composite Aerogel by Hydrothermal Method and Its Adsorption Property of Methyl Orange, Polymers. $12 \quad$ (2020) 2169. doi:10.3390/polym12092169.

[25] N.L. Zaabaa,K. .L.Fooa,K.L.Hashima, U.;Tanb,S.JWen Liua,Wei.Voona, Synthesis of Graphene Oxide using Modified Hummers Method SolventInfluence. Advances in Materials \& Processing Technologies Conference, 2017,doi: 10.1016/j.proeng.2017.04.118

[26] I.S. Ryu, X. Liu, YJin, Y.Sun, J. Lee, StoOichiometric analysis of competing intermolecular hydrogen bonds using infrared spectroscopy, RSC Advances. 8(42) (2018) 23481-23488. doi:10.1039/c8ra02919a.

[27] J.Bonilla, E. Fortunati ,L.Atarés, A.Chiralt, A.;I.M. Kenny, J. M. L, Physical, structural and antimicrobial properties of poly vinyl alcohol-chitosan biodegradable films, Food Hydrocolloids.35 (2014) 463-470. doi:10.1016/j.foodhyd.2013.07.00

[28] Matica, Aachmann, Tфndervik, Sletta, Ostafe, Chitosan as a Wound Dressing Starting Material Antimicrobial Properties and Mode of Action, International Journal of Molecular Sciences. 20(23) (2019) 5889. doi:10.3390/ijms20235889

[29] S. Kim, Competitive Biological Activities of Chitosan and Its Derivatives, Antimicrobial, Antioxidant, Anticancer, and Anti-Inflammatory Activities, International Journal of Polymer Science. (2018) 1-13. doi:10.1155/2018/17081. 